\title{
Modeling Simple-Cell Direction Selectivity With Normalized, Half-Squared, Linear Operators
}

\author{
DAVID J. HEEGER \\ Department of Psychology, Stanford University, Stanford, California 94305
}

\section{SUMMARY AND CONCLUSIONS}

1. A longstanding view of simple cells is that they sum their inputs linearly. However, the linear model falls short of a complete account of simple-cell direction selectivity. We have developed a nonlinear model of simple-cell responses (hereafter referred to as the normalization model) to explain a larger body of physiological data.

2. The normalization model consists of an underlying linear stage along with two additional nonlinear stages. The first is a half-squaring nonlinearity; half-squaring is half-wave rectification followed by squaring. The second is a divisive normalization nonlinearity in which each model cell is suppressed by the pooled activity of a large number of cells.

3 . By comparing responses with counterphase (flickering) gratings and drifting gratings, researchers have demonstrated that there is a nonlinear contribution to simple-cell responses. Specifically they found $I$ ) that the linear prediction from counterphase grating responses underestimates a direction index computed from drifting grating responses, 2) that the linear prediction correctly estimates responses to gratings drifting in the preferred direction, and 3) that the linear prediction overestimates responses to gratings drifting in the nonpreferred direction.

4. We have simulated model cell responses and derived mathematical expressions to demonstrate that the normalization model accounts for this empirical data. Specifically the model behaves as follows. 1) The linear prediction from counterphase data underestimates the direction index computed from drifting grating responses. 2) The linear prediction from counterphase data overestimates the response to gratings drifting in the nonpreferred direction. The discrepancy between the linear prediction and the actual response is greater when using higher contrast stimuli. 3) For an appropriate choice of contrast, the linear prediction from counterphase data correctly estimates the response to gratings drifting in the preferred direction. For higher contrasts the linear prediction overestimates the actual response, and for lower contrasts the linear prediction underestimates the actual response.

5 . In addition, the normalization model is qualitatively consistent with data on the dynamics of simple-cell responses. Tolhurst et al. found that simple cells respond with an initial transient burst of activity when a stimulus first appears. The normalization model behaves similarly; it takes some time after a stimulus first appears before the model cells are fully normalized. We derived the dynamics of the model and found that the transient burst of activity in model cells depends in a particular way on stimulus contrast. The burst is short for high-contrast stimuli and longer for low-contrast stimuli.

6. The importance of these results is that the normalization model preserves the essential features of linearity in the face of apparently contradictory behavior. According to the model, a cell's direction selectivity is attributed to the underlying linear stage, and a cell's nonlinear behavior is attributed to half-squaring and normalization.

\section{INTRODUCTION}

Many simple cells in cat primary visual cortex (striate cortex, area 17, V1) are direction selective, meaning that they respond vigorously to stimuli moving in a preferred direction but less well to stimuli moving in the nonpreferred (opposite) direction (Hubel and Wiesel 1962).

A longstanding view of simple cells is that they sum their inputs linearly (Campbell et al. 1968, 1969; Hubel and Wiesel 1962). The linear model is commonly used to explain a simple cell's selectivity for stimulus orientation and spatial frequency. Recently, researchers have proposed linear models to explain direction selectivity as well (Adelson and Bergen 1985; Burr et al. 1986; Fahle and Poggio 1981; Watson and Ahumada 1983, 1985). The linear model is an attractive explanation because the response of a linear cell can be completely characterized with a relatively small number of measurements.

The response of a linear cell is a weighted sum (over local space and recently past time) of the intensity values in the stimulus. Direction selectivity arises because of suitable timing differences (delays) in the responses evoked from different parts of the cell's receptive field.

Figure $1 \mathrm{~A}$ illustrates a simple example of a vertical bar moving to the right over time. Imagine that we film a movie of this stimulus and stack the consecutive frames one after the next. This yields a three-dimensional volume (spacetime cube) of intensity data like that shown in Fig. $1 B$. Figure $1 C$ shows an $x$ - $t$ slice through this space-time cube. The slope of the edges in the $x-t$ slice equals the horizontal component of the bar's velocity (change in position over time). Different speeds correspond to different slopes.

Figure $2 A$ illustrates a direction-selective, linear cell responding to a rightward drifting bar stimulus. Each panel in Fig. $2 A$ corresponds to a different (present) time. The left panel of Fig. $2 A$ shows the stimulus superimposed over an inhibitory subregion of the cell's space-time weighting function. The middle panel shows the situation some time later. Now the stimulus lines up with an excitatory subregion. The right panel shows the situation later still when the stimulus again lines up with an inhibitory subregion. Figure $2 B$ is a plot of the cell's response over time. There is a vigorous modulated response as the bar passes through the cell's receptive field.

Figure 2, $C$ and $D$, illustrate the same linear cell responding to a bar drifting in the nonpreferred direction of motion. In this case there is very little response. The bar simultaneously stimulates all three subregions, and the excitation and inhibition cancel one another. 


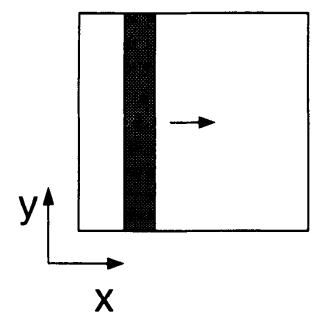

A

There are some empirical results that are consistent with the spatiotemporal linear model of direction selectivity. First, Dean and Tolhurst (1986) have measured timing differences between different receptive-field subregions.

Second, McLean and Palmer (1989), Shapley et al. (1991), and DeAngelis et al. (1993a,b) have measured the spatiotemporal weighting functions of simple cells with the

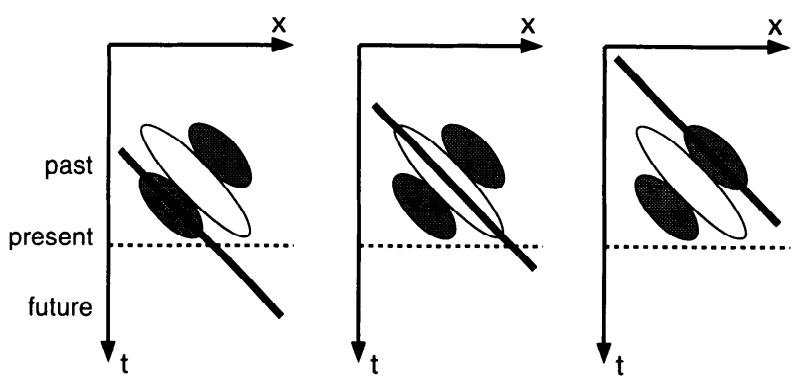

A
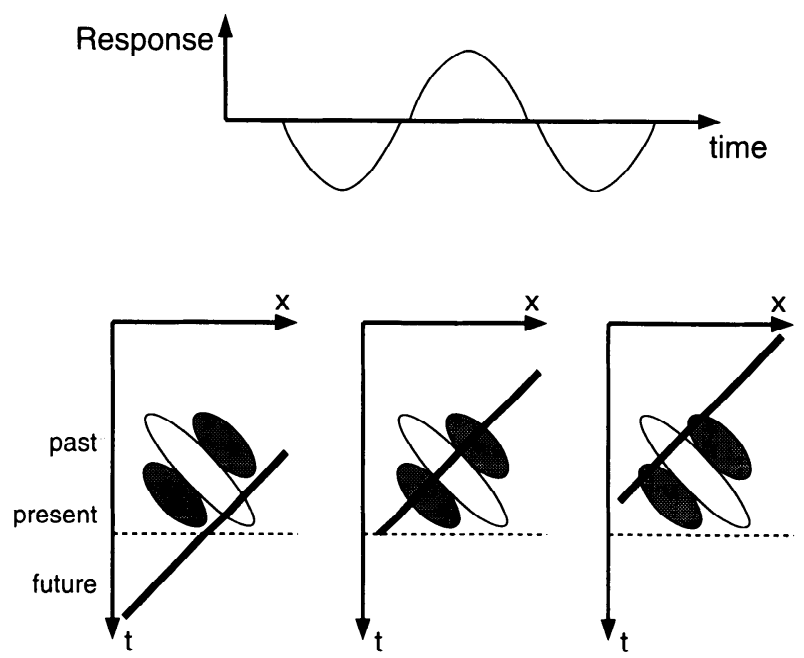

C

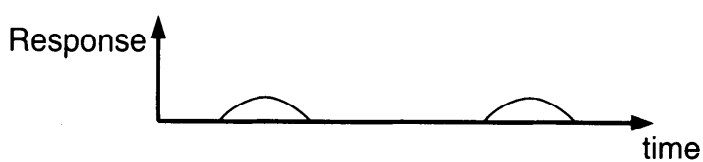

FIG. 2. Illustration of a spatiotemporal linear cell responding to drifting bar stimuli. $A$ and $B$ : bar drifting in preferred direction. $C$ and $D$ : bar drifting in the nonpreferred direction. $A$ and $C$ : snapshots of stimuli superimposed on linear weighting function. Stimuli depicted as obliquely oriented lines in the $x-t$ diagram. Linear weighting function is drawn by the use of obliquely oriented ellipses. Shaded ellipses correspond to inhibitory spatiotemporal subregions of the space-time weighting function, and the unshaded ellipse corresponds to the excitatory subregion. $B$ and $D$ : plots of response over time as the bar drifts through the receptive field. use of white-noise stimuli. They found some simple cells with weighting functions tilted along an oblique axis in space-time, like that illustrated in Fig. 2. The linear model predicts that these cells be direction selective. In fact, because a spatiotemporal linear cell is completely characterized by its spatiotemporal weighting function, the model allows one to predict a cell's preferred direction and speed of motion from its weighting function. McLean and Palmer (1989) tested this and found that they could, for most cells, correctly predict the preferred bar motion from the spatiotemporal weighting function.

However, recent experiments (Albrecht and Geisler 1991; Reid et al. 1987, 1991; Tolhurst and Dean 1991) have demonstrated that the linear model fails to account for certain empirical findings. These experiments compared responses of simple cells with drifting and counterphase flickering (stationary, temporally modulated) sinusoidal gratings. A counterphase grating stimulus can be expressed as the sum of two drifting gratings, drifting in opposite directions. For a linear cell the response to the sum of two stimuli is equal to the sum of the responses to each stimulus presented alone. Thus one can easily predict counterphase grating responses from the drifting gratings responses, and vice versa. For simple cells, however, these linear predictions systematically fail (see Figs. 9 and 10).

The goal of this paper is to explain why these linear predictions fail and to offer an alternative model (actually, an extension of the linear model) that is consistent with the data. The new model is based on the linear model, but it includes two additional nonlinear stages. First, model simple-cell responses are half-squared (half-squaring is halfwave rectification followed by squaring). Second, each model cell is normalized by the pooled activity in a large number of cells.

Some of the components of this normalization model have been proposed previously (Albrecht and Geisler 1991; Carandini and Heeger 1993; Heeger and Adelson 1989; Heeger 1990, 1991, 1992a-c; Robson 1988). Indeed, previous work on the normalization model (Carandini and Heeger 1993; Heeger 1991, 1992a,b) has already demonstrated that it explains a significant body of physiological data. In particular, the normalization model has been used to simulate physiological measurements of response versus contrast, contrast adaptation, and nonspecific suppression. None of these experimental results are consistent with the linear model, but they are all qualitatively consistent with the normalization model.

This paper demonstrates that the new model, with half- 


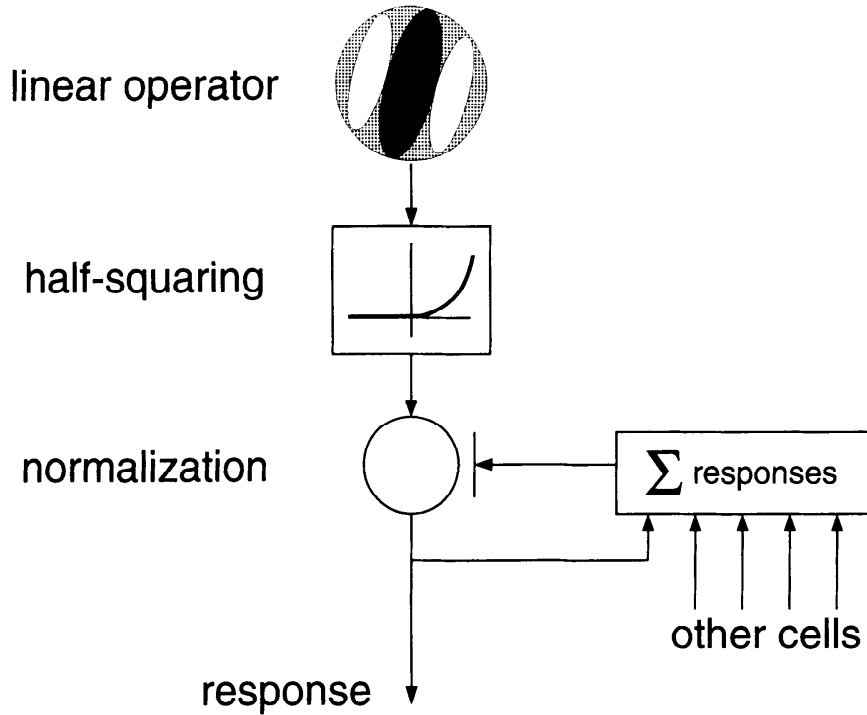

FIG. 3. Illustration of the 3 stages in the model: 1 ) an underlying linear stage that pools its inputs with the use of addition and subtraction; 2) a half-squaring nonlinearity (half-squaring is half-wave rectification followed by squaring); and 3) a divisive normalization nonlinearity in which each model cell is suppressed by the pooled activity of a large number of cells.

squaring and normalization, accounts for the aforementioned empirical results (Albrecht and Geisler 1991; Reid et al. 1987, 1991; Tolhurst and Dean 1991) that revealed nonlinearities in simple-cell direction selectivity. The importance of this result is that the new model preserves the essential features of linearity in the face of apparently contradictory behavior.

\section{METHODS}

This section reviews the basic components of the new model. As illustrated in Fig. 3, the model has three stages: 1) an underlying linear stage that pools its inputs with the use of addition and subtraction; 2) a half-squaring nonlinearity (half-squaring is halfwave rectification followed by squaring); and 3 ) a divisive normalization nonlinearity. In other words, simple cells are characterized as normalized, half-squared, linear operators. The normalization is implemented via feedback in which each model cell is suppressed by the pooled activity in a large number of cells. APPENDIX A gives further details on this feedback normalization network.

\section{Linear operators}

The response of a linear operator (a linear cell) is expressed as a weighted sum, over local space and recently past time, of the stimulus intensities. The response $L(t)$ is the inner product in space and the convolution in time of a stimulus $I(x, y, t)$ with the operator's spatiotemporal weighting function $f(x, y, t)$

$$
L(t)=\iiint_{-\infty}^{\infty} f(x, y, \tau) I(x, y, \tau-t) \mathrm{d} x \mathrm{~d} y \mathrm{~d} \tau
$$

The triple integral in the above equation is simply a weighted sum of the stimulus intensities over space and time. The response $L(t)$ is the model equivalent of a poststimulus time histogram (PSTH), a measure of an operator's average response per unit time. The structure of the spatiotemporal weighting function $f(x, y, t)$ determines the operator's selectivity (e.g., for orientation, direction of motion, and spatial and temporal frequency).

The linear weighting functions considered in this paper have positive and negative subregions. The positive and negative weights are balanced, so the model cells do not respond to stimuli with constant intensity. Rather, their responses are proportional to stimulus contrast, for stimuli that vary in intensity over space and/or time.

\section{Half-squaring}

Cell responses (firing rates) are by definition positive, whereas linear operators can have positive or negative responses. A linear cell with a high maintained firing rate could encode the positive and negative values by responding either more or less than the maintained rate. Simple cells, however, have very little maintained discharge so they cannot truly act as linear operators.

The positive and negative values can rather be encoded by two cells: one responsible for the positive part and the other one responsible for the negative part. The two cells are complements of one another. That is, an excitatory subregion of one cell's receptive field is aligned with an inhibitory subregion of the other cell's receptive field. The response of each cell is half-wave rectified so that only one of the two cells has a nonzero response at any given time.

A number of researchers have characterized simple cells as rectified linear operators (e.g., Movshon et al. 1978). Variants of this characterization have used different types of rectification. For example, overrectification is like half-wave rectification but with a threshold; the neuron has to reach a certain level of excitation before it will fire action potentials.

The normalization model advocated in this paper uses halfsquaring as an alternative form for the rectification. Half-squaring is half-wave rectification followed by squaring

$$
A(t)=\lfloor L(t)\rfloor^{2}
$$

where $A(t)$ is the response of a half-squared, linear operator, [x] $=$ $\max (x, 0)$ is half-wave rectification, and $L(t)$ is the linear response defined in $E q .1$.

\section{Normalization}

Normalization in the model works by suppressing each cell's response by the pooled activity of a large number of cells. Consider a collection of linear operators (i.e., a collection of cells), with various receptive-field centers (covering the visual field) and with various spatiotemporal frequency, orientation, and direction tunings. Let $A_{i}(t)$ denote the response of an unnormalized, halfsquared, linear operator. The normalized response $R_{i}(t)$ is defined as

$$
R_{i}(t)=K \frac{A_{i}(t)}{\sigma^{2}+\sum_{j} A_{j}(t)}
$$

where $\sigma^{2}$ is the semisaturation constant, $K$ determines the maximum attainable response, and the summation in the denominator $\sum_{j} A_{j}(t)$ is taken over a large number of operators with different tunings. This summation $\sum_{j} A_{j}(t)$ includes the term $A_{i}(t)$ that appears in the numerator (i.e., each cell suppresses itself). As long as $\sigma$ is nonzero, the normalized response will always be a value between 0 and $K$, saturating for high contrasts.

The underlying linear weighting functions can be chosen so that they tile the spatiotemporal frequency domain, i.e., the sum of their power spectra is a constant function (everywhere equal to a constant value). Thus the normalization signal, the pooled activity of many cells, is proportional to the integral of the power spectrum (the Fourier energy) of the stimulus. ${ }^{1}$

\footnotetext{
"The normalization signal can also be computed "locally" by pooling responses of cells over a limited region of space and a limited range of spatiotemporal frequency tunings. The normalization signal is then proportional to the "local" Fourier energy of the stimulus. For grating stimuli, the local normalization signal is reasonably well approximated by the total
} 
The conclusions of this paper depend critically on the fact that the normalization signal is proportional to the Fourier energy of the stimulus. Fourier energy is not the same as contrast. In particular, drifting gratings and counterphase gratings of equal Michelson contrast have different Fourier energies. A drifting grating with contrast $c$ has Fourier energy equal to $c^{2}$. A counterphase grating with contrast $c$ is made up of two drifting gratings, each with contrast $(c / 2)$, drifting in opposite directions. The Fourier energy of the counterphase grating is therefore equal to $(c / 2)^{2}+(c / 2)^{2}=$ $c^{2} / 2$. This difference between the Fourier energies of the two stimuli is the key to explaining the empirical data on direction selectivity (see RESULTS).

Normalization in the model is actually performed by a feedback network of interconnections. Details of this feedback normalization network are given in APPENDIX A. The feedback normalization network is designed so that for drifting grating stimuli, the network's steady-state behavior is given by $E q$. 3 .

One consequence of using a feedback network to achieve the normalization is that the feedback signal (the pooled responses) must $b c$ averaged over time to avoid unstable oscillations. It thus takes some time after a stimulus first appears before the model cells are fully normalized. Consequently, there is an initial transient burst of activity in model cell responses (see Fig. 5).

\section{Detailed methods}

A particular set of model cells was used to compute the simulation results presented below. Each model cell is tuned to one of four orientations (separated by $45^{\circ}$ ) and one of five spatial frequencies (with octave bandwidth and octave spacing). For each spatial frequency and orientation there are three temporal channels, one preferring static stimuli and the other two preferring moving stimuli (e.g., vertical static, vertical rightward, and vertical leftward). For each spatial frequency, orientation, and temporal band there are cells with four different phases, in $90^{\circ}$ steps. In total there are 240 cells in the model.

The model cells were designed in quadruples, four cells with the same amplitude response but with phases in steps of $90^{\circ}$. Each of the four is half-squared. For a drifting sine grating stimulus, the sum of the four responses is proportional to the squared contrast of the stimulus, and it is constant over time (independent of the stimulus phase). The phase independence is a simple consequence of the $90^{\circ}$ phase offsets, analogous to the trigonometric identity: $\sin ^{2}(\theta)+\cos ^{2}(\theta)=1$. It is important that responses can be summed to give a time-invariant signal, so that the model's behavior can be analyzed analytically ( see APPENDIX A).

The linear weighting functions of the model cells were designed so that cach is a spatial and/or temporal derivative a particular function $f(x, y, t)$. The function $f(x, y, t)$ was chosen so that the full set of (derivative) weighting functions evenly cover (tile) the frequency domain, i.e., so that the sum of their squared responses is proportional to the Fourier energy of the stimulus.

For example, the weighting function of one cell is the third derivative of $f(x, y, t)$ in the horizontal direction $f_{x x x}(x, y, t)$. The weighting function of another is the third derivative in the vertical direction $f_{y y y}(x, y, t)$. These two cells are nondirection selective. The former prefers static vertical stimuli, and the latter prefers static horizontal stimuli.

The direction-selective weighting functions were constructed by summing two nondirection-selective (space-time separable) subunits. For example, the weighting function preferring leftward vertical stimuli is $f_{x x x}+f_{x x t}$.

Figure 4 shows space-time slices through three space-time

Fourier energy. This is a particularly good approximation when the normalization is pooled over a region of space and frequency that is considerably larger than the summation region of the underlying linear weighting function. weighting functions: $f_{x x x}, f_{x x t}$, and $f_{x x x}+f_{x x t}$. Although $f_{x x x}$ and $f_{x x t}$ are each space-time separable (nondirection selective), their sum is tilted in space-time (direction selective). All of the cells in the model have temporal responses that are either monophasic (like $f_{x x x}$ ) or biphasic (like $\left.f_{x x x}+f_{x x t}\right)$.

Watson and Ahumada (1983, 1985) and Adelson and Bergen (1985) proposed a similar procedure for constructing direction-selective weighting functions, by summing two space-time separable subunits. Their subunits are related to one another by a quadrature phase shift both in space and in time.

The weighting functions used in this paper have slightly different properties. Although they are constructed by summing two space-time separable subunits, the subunits are not quadrature pairs. Rather, as discussed above, they are all spatial and/or temporal derivatives of the same function $f(x, y, t)$.

The choices for the model cells' spatiotemporal frequency tunings and bandwidths are arbitrary from a theoretical point of view, and the conclusions of this paper do not depend of these particular choices. The tunings and bandwidths are, however, in rough agreement with those of typical simple cells.

Each model cell is normalized by the pooled activity of a large number of cells. In the present implementation of the model, the normalization signal is pooled over three spatial frequency bands and over all three temporal bands. The normalization signal is thus the Fourier energy of the stimulus in a three-octave wide annulus of spatiotemporal frequencies. Model cells that differ only in their orientation tuning are all normalized by the same factor. Model cells that differ only in their temporal frequency tuning are also normalized by the same factor. Model cells tuned to different spatial frequencies are normalized by different factors, that is, by different annuli of spatiotemporal frequencies. The temporal averaging of the normalization signal is discussed in APPENDIX A. The spatial averaging of the normalization signal is left unspecified in this paper for the sake of simplicity. The spatial pooling of the normalization was unimportant because the stimuli were all spatially extended gratings.

There are three additional parameters of the model: $\sigma$ is the semisaturation constant, $\alpha$ specifies the temporal averaging of the feedback ( see APPENDIX A for details), and $K$ determines the maximum attainable response. These three parameters were chosen to have the following values: $K=1, \sigma=0.1$, and $\alpha=0.01$. For most of the model simulations, the stimulus contrast was $20 \%$.

The model simulations were computed as follows. First, the normalized, half-squared responses were computed by the use of the feedback normalization network. Then, response amplitudes (proportional to the peak response) and response phases (relative peak latency) were computed as the magnitude and phase of the fundamental Fourier component of each normalized response. The first quarter-second of the normalized responses were ignored when computing response amplitude and phase, thereby avoiding the initial transient behavior of the feedback network. Note that the magnitude and phase of the fundamental does not completely characterize the responses because they are not sinusoidal (see Fig. 5 ). Even so, computing response amplitude and response phase allows a direct comparison with published physiological results.

The simulated responses were computed for drifting and counterphase grating stimuli of various spatial and temporal frequencies. For drifting gratings, response amplitudes were obtained for both the preferred and nonpreferred directions of motion.

For counterphase gratings, responses were simulated for eight stimulus spatial phases at intervals of $22.5^{\circ}$. Response amplitudes and response phases were obtained for each of the eight stimulus phases. Polar plots of response amplitude as a function of response phase were roughly elliptical in shape (see Fig. 8). An ellipse was fit to these counterphase grating responses, with the use of the fitting procedure described by Reid et al. (1991).

There were, in summary, four simulated measurements for each spatial and temporal frequency: 1) $R_{\mathrm{p}}$ is the response to a 


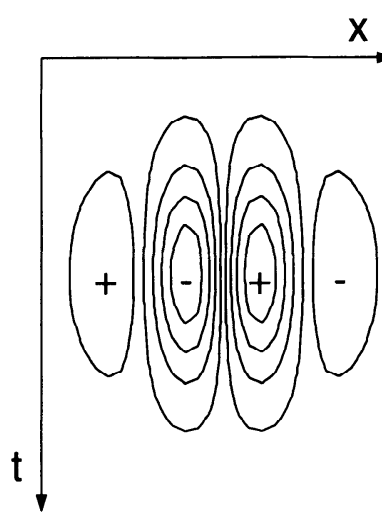

A

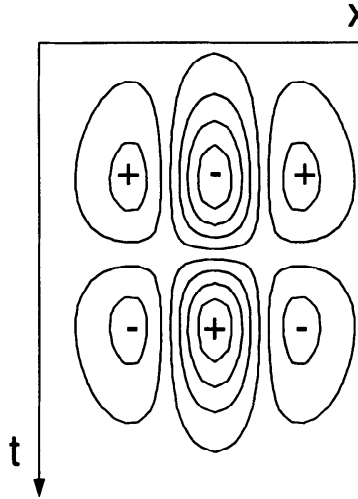

B

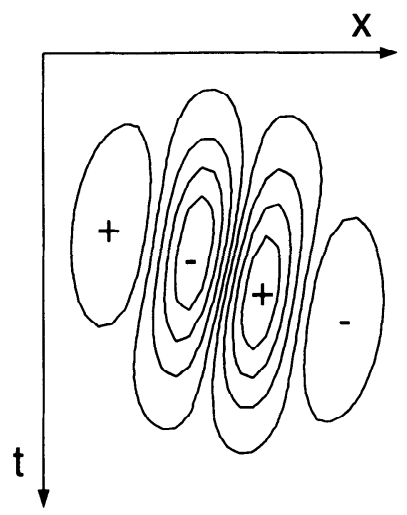

C
FIG. 4. Space-time slices of linear weighting functions designed so that each is a spatial and / or temporal derivative of a particular function, $f(x, y, t) . A: f_{x x x}$, the 3 rd spatial derivative, is monophasic in time and space-time separable. Cells with this weighting function prefer static vertical stimuli. $B: f_{x x t}$ is biphasic in time and space-time scparable. Cells with this weighting function prefer flickering vertical stimuli. $C:\left(f_{x x x}+\right.$ $f_{x x t}$ ) is tilted in space-time (not space-time separable). Cells with this weighting function prefer leftward motion. grating drifting in the preferred direction, 2) $R_{\mathrm{n}}$ is the response to a grating drifting in the nonpreferred direction, 3 ) $R_{1}$ is the response to a counterphase grating with the spatial phase that elicits the largest response (i.e., the major axis of the ellipse), and 4) $R_{2}$ is response to a counterphase grating that elicits the smallest response (i.e., the minor axis of the ellipse). These four measurements were repeated for a range of spatial and temporal frequencies.

\section{RESULTS}

This section reviews experimental measurements of simple-cell direction selectivity and compares simulated responses of model cells with physiological data. No attempt was made to fit model parameters to the data, so the simulations merely point out the qualitative similarities between the behavior of model cells and real cells.

\section{Drifting grating responses}

Simplc cells exhibit characteristic responses to drifting sine gratings. This section shows that model simple cells (normalized, half-squared, and linear operators) respond similarly.

The response of a linear cell to a drifting grating varies sinusoidally over time with the same temporal frequency as that of the stimulus. Real simple cells, however, respond over approximately one-half of each stimulus cycle, remaining silent during the other half-cycle (Andrews and Pollen 1979; Kulikowski and Bishop 1981; Movshon et al. 1978).

Figure 5 shows that a model cell also responds over only one-half of each cycle. Figure $5 \mathrm{~A}$ plots the simulated response of a typical model cell to a sinusoidal grating drifting in the cell's preferred direction. Figure $5 B$ shows the simulated response to a grating drifting in the nonpreferred direction. Direction selectivity is evident because the amplitude of the response is greater in Fig. $5 A$ than in Fig. $5 B$.

Figure 6 shows how direction selectivity is affected by stimulus contrast. Figure $6 \mathrm{~A}$ plots the response of a simple cell as a function of stimulus contrast for gratings drifting in both the preferred and nonpreferred directions. Figure $6 B$ plots the simulated responses for a typical model cell. The simulated curves saturate (because of normalization) at high stimulus contrasts, in a manner quite similar to real cell behavior.

In addition, for both real cells and model cells the curve shifts downward (on the log scale) for motion in the non- preferred direction. ${ }^{2}$ In other words, direction selectivity (defined here as the ratio of the responses produced by the 2 different stimuli) is largely invariant with respect to stimulus contrast, in spite of saturation. This invariance is due to normalization in the model, and it is critical for encoding information about motion independent of contrast (Heeger 1992a).

\section{Counterphase grating responses}

Simple cells exhibit characteristic responses to counterphase flickering sine gratings. The response varies over time with the temporal modulation of the stimulus, and the amplitude and phase of modulation both depend on the spatial phase of the grating (Kulikowski and Bishop 1981; Maffei and Fiorentini 1973; Movshon et al. 1978; Reid et al. 1987). This section points out that a model cell responds similarly.

Movshon et al. (1978), Reid et al. (1987, 1991), and Tolhurst and Dean (1991) measured response amplitude and response phase of simple cells while varying the spatial phase of counterphase gratings. They have shown that for a linear cell a polar plot of the response amplitude as a function of the response phase is elliptical in shape. Their experimental results, however, are typically not quite elliptical. Rather the results are described as "wasp-waisted ellipses" because the amplitudes near the minor axes are smaller than they should be to fit an ellipse. An example, replotted from Tolhurst and Dean (1991), is shown in Fig. 7.

Movshon et al. (1978) proposed that the deviation from an ellipse could be explained by overrectification. If the neuron has to reach a certain level of excitation before any activity is seen, there will be a disproportionate decrease in small responses.

The wasp-waisted elliptical shape is also predicted by half-squaring and normalization. Figure 8 shows waspwaisted results from a typical model cell for stimuli of several

\footnotetext{
${ }^{2}$ Similar downward shifts of response-versus-contrast curves have been measured for stimuli of nonpreferred direction of motion, nonoptimal orientation, nonoptimal spatial or temporal frequency, and for stimuli in the nondominant eye (Albrecht and Hamilton 1982; Dean 1980, 1981; Holub and Morton-Gibson 1981; Li and Creutzfeldt 1984; Sclar and Freeman 1982). Some have interpreted these results as demonstrating that saturation of the contrast-response curve is already present at the precortical level and therefore not because of intracortical mechanisms. On the contrary, the simulated results in Fig. $6 B$ exhibit this downward shift precisely because of mutual suppression between cortical cells (Heeger 1992a).
} 

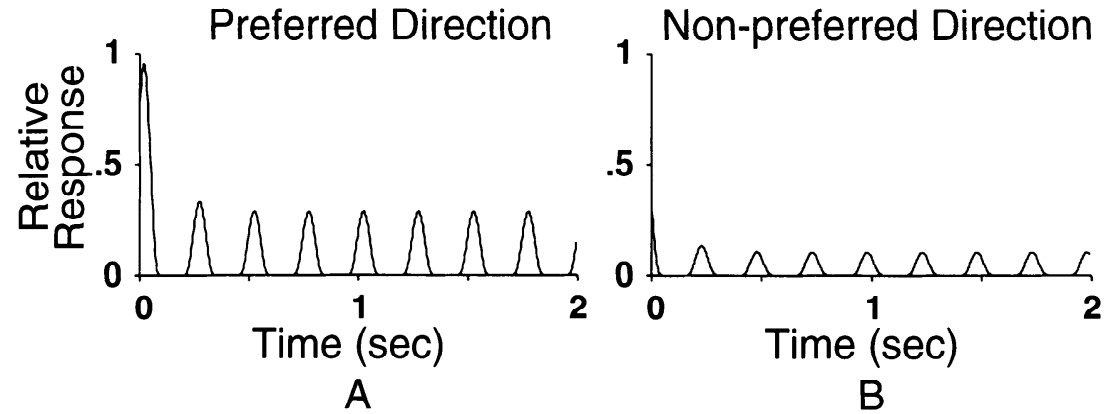

FIG. 5. $A$ : response of model cell to sinusoidal grating $(20 \%$ contrast, preferred spatial frequency and orientation) drifting in preferred dircction. Initial transient burst of activity (evident during the 1st cycle of the response) is due to feedback normalization (see APPENDIX A for details). $B$ : response to grating drifting in the nonpreferred direction. different temporal frequencies. Note that some ellipses show more of a wasp-waist than others. Note also that the ellipses become more elongated (less direction selective) at high temporal frequencies. Both of these results are typical of real simple cells (see Fig. 5 of Reid et al. 1991). The elongation of the ellipses is due to the design of the underlying linear weighting functions (see Detailed methods). There are some direction-selective weighting functions for which this elongation would not occur.

\section{Drifting gratings versus counterphase gratings}

By comparing the responses to counterphase gratings with responses to drifting gratings, Reid et al. (1987, 1991), Tolhurst and Dean (1991), and Albrecht and Geisler (1991) found that there is a nonlinear contribution to simple-cell responses. This section explains that this nonlinear behavior can be attributed to half-squaring and normalization. The importance of this result is that the normalization model preserves the essential features of linear motion mechanisms in the face of apparently contradictory behavior.

Reid et al. (1987, 1991) showed that for a linear cell the responses to drifting gratings are predictable from the responses to counterphase gratings (of the same spatial and temporal frequency as the drifting gratings). Specifically, the response to gratings drifting in the preferred and nonpreferred directions are predictable from the axes of the ellipse obtained, as described above, from counterphase gratings

$$
\begin{aligned}
& R_{\mathrm{p}}=R_{1}+R_{2} \\
& R_{\mathrm{n}}=R_{1}-R_{2}
\end{aligned}
$$

Following Reid et al. (1987, 1991) we use a direction index to summarize the direction selectivity of a cell. The direction index $D I$ is defined as

$$
D I=\left(R_{\mathrm{p}}-R_{\mathrm{n}}\right) /\left(R_{\mathrm{p}}+R_{\mathrm{n}}\right)
$$

For model cells, this direction index is invariant with respect to stimulus contrast. ${ }^{3}$ The direction index does, however, depend on the spatial and temporal frequencies of the stimulus.

From Eqs. 4-6 it follows that the direction index (measured with drifting gratings) is also predictable from counterphase grating responses

\footnotetext{
${ }^{3}$ For model cells, the direction index is invariant with respect to contrast. Tolhurst and Dean (1991), however, report measurements from one cell with a direction index that is not the same at all contrasts. At the lower contrasts, the measured direction index is somewhat greater.
}

$$
D I=R_{2} / R_{1}
$$

Reid et al. (1987, 1991), Tolhurst and Dean (1991), and Albrecht and Geisler (1991) found systematic failures of two of these three linear predictions. First, the linear predictions from counterphase grating responses underestimate the direction index computed from drifting grating responses (Fig. 9A). Second, the counterphase data overestimate responses to gratings drifting in the nonpreferred direction (Fig. 10A). Even so, the counterphase data correctly estimate responses to gratings drifting in the preferred direction (Fig. 11 $A$ ).

The half-squared, normalized, linear model behaves similarly, as shown in Figs. $9 \mathrm{~B}, 10 \mathrm{~B}$, and $11 \mathrm{~B}$. APPENDIX B gives a formal derivation of the model's behavior. An intuitive explanation is provided here.

The first of these three results ( underestimating the direction index) is entirely due to half-squaring in the model. Normalization is totally irrelevant when calculating the direction index. Because the direction index is a ratio of responses, the normalization factors in numerator and denominator exactly cancel one another.

Including normalization in the model is critical for obtaining the other two results. The normalization signal (the summation in the denominator of $E q .3$ ) is proportional to the Fourier energy of the stimulus. Drifting gratings and counterphase gratings of equal contrast have different Fourier energies. A drifting grating with Michelson contrast $c$ has Fourier energy equal to $c^{2}$. A counterphase grating with contrast $c$ has Fourier energy equal to $c^{2} / 2$. The suppression in the case of the drifting grating is greater, so the response to the nonpreferred direction is smaller than that predicted from counterphase data ( formal derivation).

\section{Effect of contrast}

Figure $12 B$ shows the simulated response to gratings drifting in the nonpreferred direction, as a function of contrast. Figure $12 \mathrm{~B}$ also shows the counterphase grating prediction as a function of contrast. For all contrasts, the counterphase prediction overestimates the actual response. The discrepancy is greater at higher contrasts.

It is interesting to note in this context that Tolhurst and Dean ( 1991) and Reid et al. (1991) used different stimulus contrasts in their experiments. Tolhurst and Dean typically used 50\% contrast gratings, and Reid et al. typically used $20 \%$. Tolhurst and Dean measured a somewhat greater discrepancy between the counterphase prediction and the response to stimuli of the nonpreferred direction.

Figure $12 \mathrm{~A}$ shows a similar plot but for the preferred direction. For low contrasts the counterphase grating pre- 


\section{Tolhurst \& Dean}
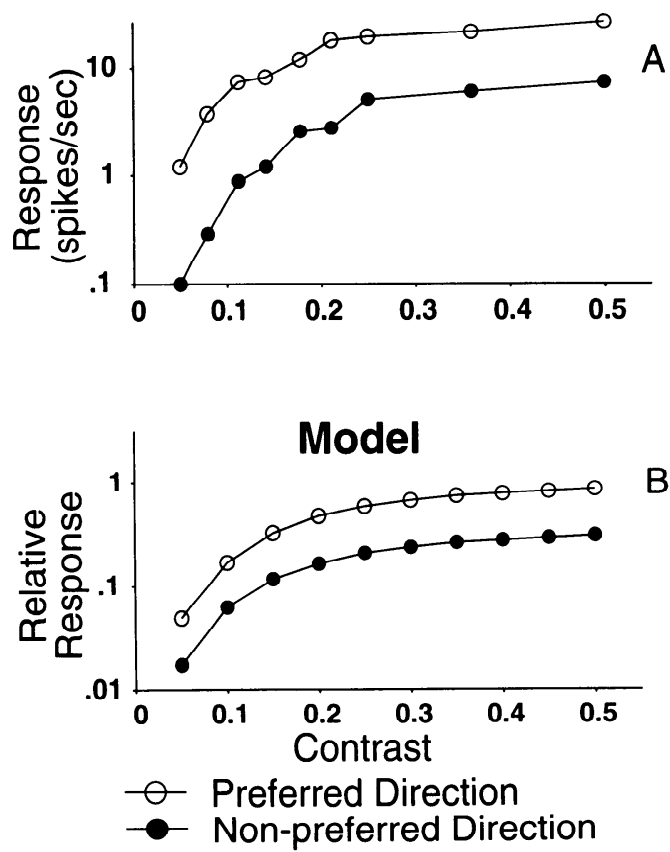

FIG. 6. $A$ : simple-cell response vs. grating contrast for grating stimuli drifting in preferred $(0)$ and nonpreferred $(\bullet)$ directions (data replotted from Tolhurst and Dean 1991). In this and subsequent figures, the plotted values are proportional to the magnitude of fundamental Fourier component of the response. Curve shifts mostly downward (on the log scale) for nonpreferred direction, indicating that direction selectivity is largely invariant with respect to contrast. $B$ : response vs. contrast for direction-selective model cell. Saturation of response and downward shift of the curve for nonpreferred direction resembles physiological data.

diction slightly underestimates the preferred response. For high contrasts, the counterphase grating prediction overestimates the preferred response. APPENDIX B gives a formal derivation of this result. It shows that, when the contrast is relatively large, the linear prediction from counterphase data overestimates the preferred response. It also shows that, when the contrast is relatively small, the linear prediction underestimates the preferred response. For an appropriate choice of contrast, in between these two extremes, the linear prediction is close to the preferred response (as in Fig. 11).

\section{Tolhurst \& Dean}

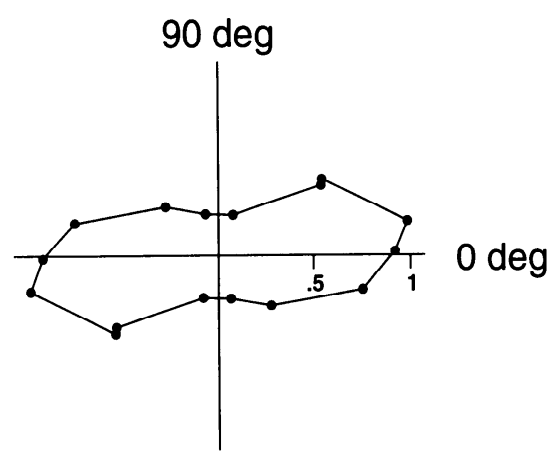

FIG. 7. Polar graph of responses to counterphase gratings (data replotted from Tolhurst and Dean 1991). Each data point represents a different stimulus spatial phase. The magnitude of the fundamental component of the response is represented radially, whereas the angular coordinate indicates the temporal phase of the response.
Discrepancy in the direction index

There is one important difference between the real data and the simulated results. For the model cells, there are stimuli (certain spatiotemporal frequencies) that are perfect nulls, i.e., the direction index $D I$ and the ellipse axis ratio $R_{2} / R_{1}$ are both equal to one. This is evident in the top right-hand corner of the graph in Fig. $9 B$. For real cells, however, the ellipse axis ratio is relatively small even when the direction index is very close to one. Although it is not obvious by comparing Fig. 9, $A$ and $B$, this discrepancy is quite significant in some of the other data sets reported by Reid et al. (1987, 1991) and by Tolhurst and Dean (1991).

To explain this difference we must first understand, for model cells, why there are certain stimuli that are perfect nulls. Figure 13 depicts a direction-selective linear cell responding to gratings drifting in both directions. In Fig. $13 B$, the space-time orientation of the stimulus is perpendicular to the preferred orientation of the cell. For any stimulus with that space-time orientation, the response will be zero (a perfect null, $D I=1$ ).

Figure $13 A$ depicts the same grating, but it is now moving in the preferred direction. The response is not zero, but at the same time the space-time orientation of the stimulus is somewhat different from the optimal space-time orientation. In other words, this is not the cell's optimal spatiotemporal frequency.

Tohurst and Dean (1991), however, only measured responses for each cell's preferred spatiotemporal frequency. They could have easily missed the $D I=1$ conditions. Reid

\section{Model}

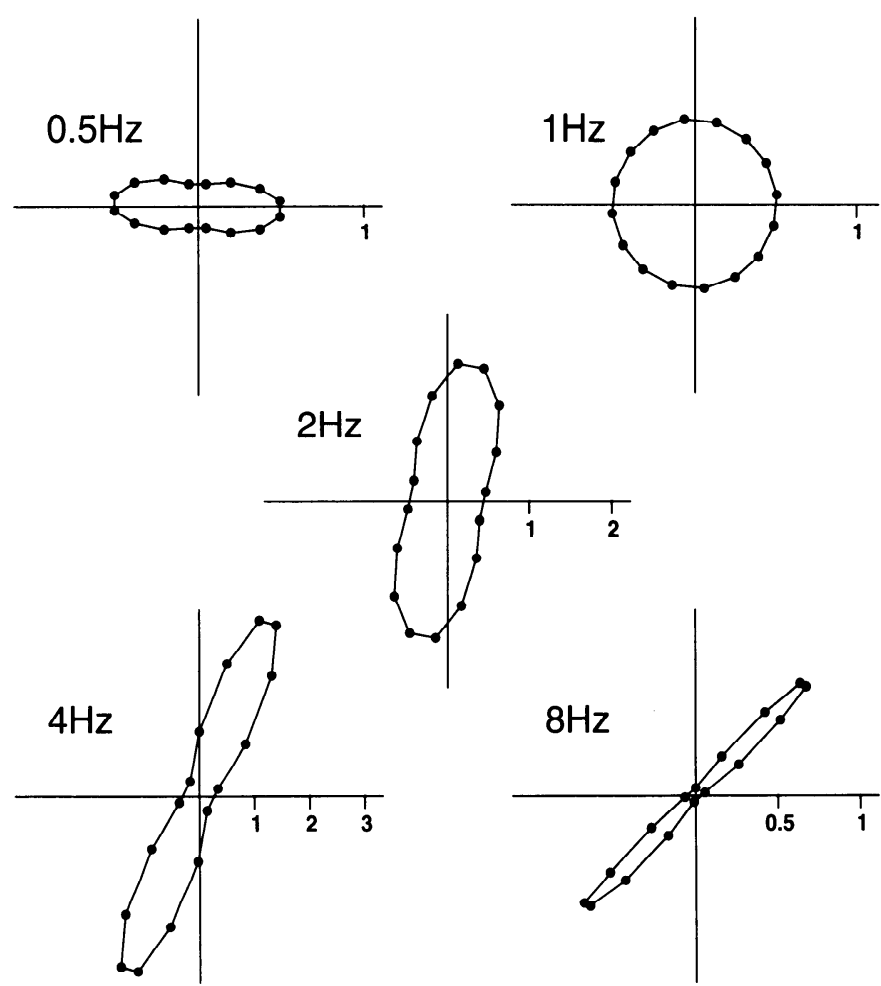

FIG. 8. Polar graphs of model cell responses to counterphase gratings. Wasp-waisted ellipses resemble the physiological data in Fig. 7. Note that the ellipses become more elongated (less direction selective) at high temporal frequencies, similar to behavior of real simple cells (see Fig. 5 of Reid et al. 1991). 
Reid, Soodak, \& Shapley

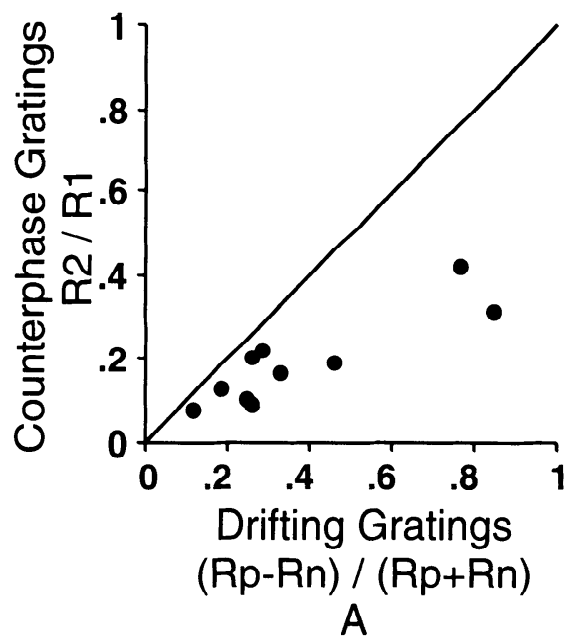

Model

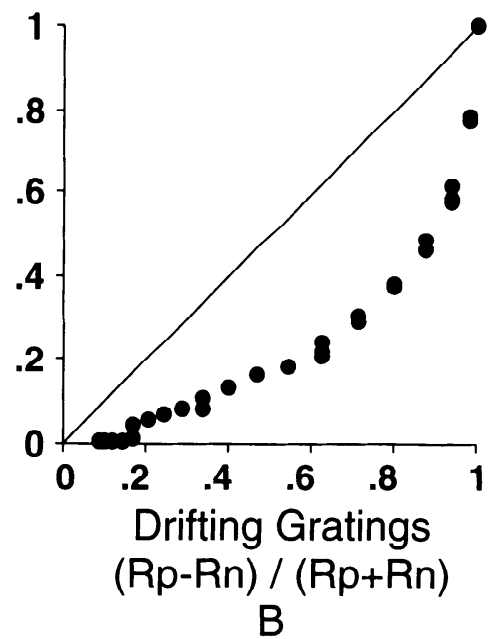

FIG. 9. Direction index predicted from counterphase data vs. that measured directly with drifting gratings. $A$ : data for a direction-selective simple cell (data provided by C. Reid). Each point is for a different stimulus spatial and temporal frequency. $B$ : simulation results for a typical model cell. For both model cells and real cells, the direction index predicted from counterphase data underestimates that measured with drifting gratings. et al. (1987, 1991) measured responses for a variety of frequencies, but they still might have missed the $D I=1$ conditions.

Moreover, there are some linear weighting functions for which the direction index will always be less than one, regardless of the stimulus frequency. Consider, for example, a linear cell with the following spatiotemporal weighting function

$$
\begin{aligned}
f(x, y, t)=e^{-\left(x^{2}+y^{2}\right)} & e^{-t^{2}} \\
& \times[\sin (2 \pi x) \cos (2 \pi t)+\beta \cos (2 \pi x) \sin (2 \pi t)]
\end{aligned}
$$

where $\beta$ is a constant. This is a direction selection weighting function that is constructed by summing two space-time separable subunits. The two units are related to one another by a quadrature phase shift both in space and in time. When $\beta=1$, the two subunits contribute equally to the direction selectivity, and there is perfect nulling $(D I=1)$ for certain stimuli. When $\beta=1 / 2$, the two subunits contribute unequally to the direction selectivity, and there are no perfect nulls ( $D I \leq 1 / 2$ for all stimuli).

The particular linear weighting functions used for the simulations in this paper were designed with other considerations in mind, so there are some frequencies that yield perfect nulls $(D I=1)$. This is not a general fault with the normalization model. Rather, it is a fault with this particular choice of linear weighting functions.

\section{DISCUSSION}

There are a variety of experimental results that reveal nonlinearities in simple-cell responses. On the basis of these results, some people have rejected the hypothesis that a cell's selectivity is due to an underlying (spatiotemporal and binocular) linear stage that pools its inputs by the use of addition and subtraction.

To the contrary, results reported in this and other papers (Albrecht and Geisler 1991; Carandini and Heeger 1993; Heeger 1991, 1992a,b) support the linear model of selectivity. According to the model advocated in these papers, a cell's selectivity is attributed to an underlying linear stage, and a cell's nonlinear behavior is attributed to half-squaring and normalization.

The normalization model appears to provide an adequate explanation of direction selectivity in simple cells. The importance of this result is that the model preserves the essential features of linearity in the face of apparently contradictory behavior.

\section{Why normalization}

It is commonly believed that information about a visual stimulus, other than its contrast, is represented as the relative responses of collections of cells. For example, the orientation of a grating might be represented as the ratio of the

\section{Reid, Soodak, \& Shapley}

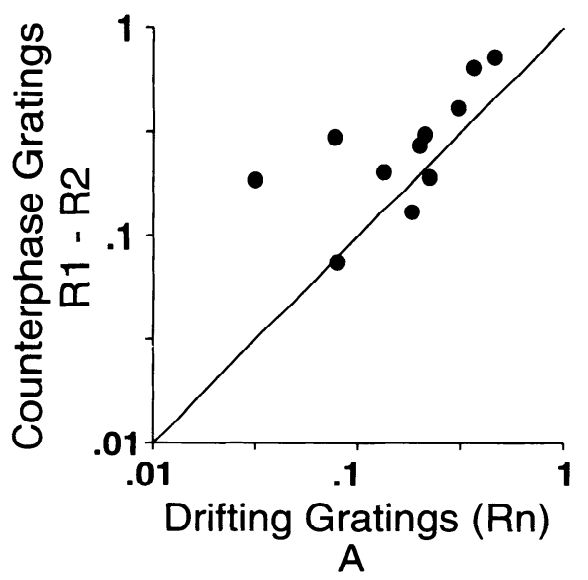

\section{Model}

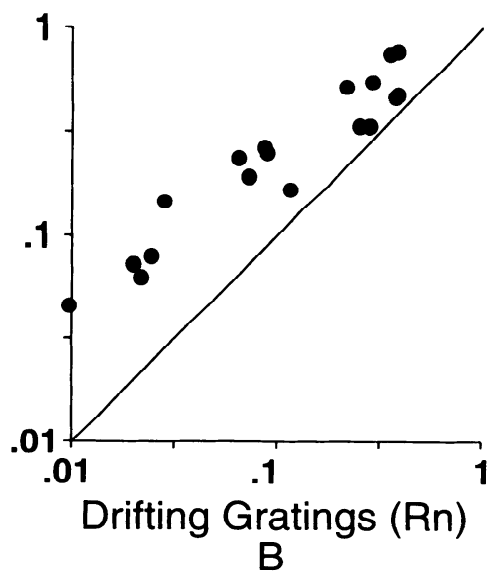

FIG. 10. Nonpreferred response predicted from counterphase data vs. that measured directly with drifting gratings. $A$ : relative responses for a directionselective simple cell (data provided by C. Reid). $B$ : simulation results for a typical model cell. For both model cells and real cells, the response predicted from counterphase data overestimates that measured directly with drifting gratings. 
Reid, Soodak, \& Shapley
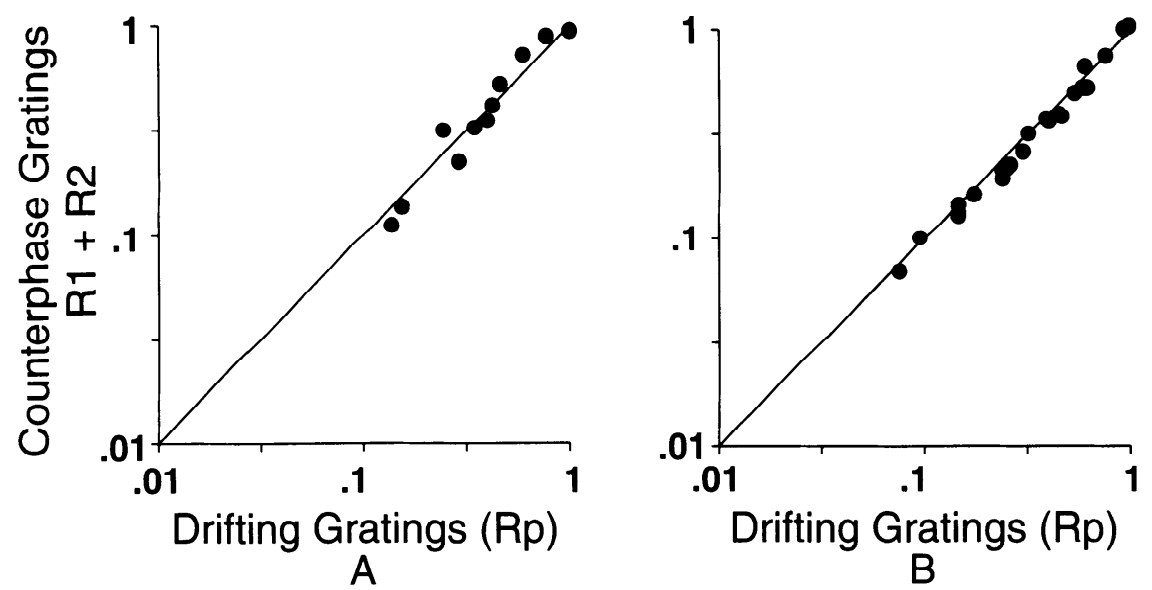

FIG. 11. Preferred response predicted from counterphase data vs. that measured directly with drifting gratings. $A$ : relative responses for a direction-selective simple cell (data provided by C. Reid). $B$ : simulation results for a typical model cell. For both model cells and real cells, the response predicted from counterphase data correctly estimates that measured directly with drifting gratings. responses of two cells, each with a different orientation preference. Indeed, physiologists have found that the ratio of a cell's responses to two stimuli is largely independent of stimulus contrast (see Fig. 6 and footnote 2). But cortical cells have a limited dynamic range, and their responses saturate for high contrasts. How is it possible for response ratios to be independent of stimulus contrast in the face of response saturation? Normalization and automatic gain control are standard engineering techniques for dealing with limited dynamic range.

There is significant empirical support for normalization in striate cortex ( see Heeger 1992a for review). Normalization can explain a wide variety of physiological data, including physiological measurements of response versus contrast, contrast adaptation, and nonspecific suppression (e.g., cross-orientation inhibition).

\section{Why squaring}

A number of researchers have characterized simple cells as rectified linear operators (e.g., Movshon et al. 1978). Variants of this characterization have used different types

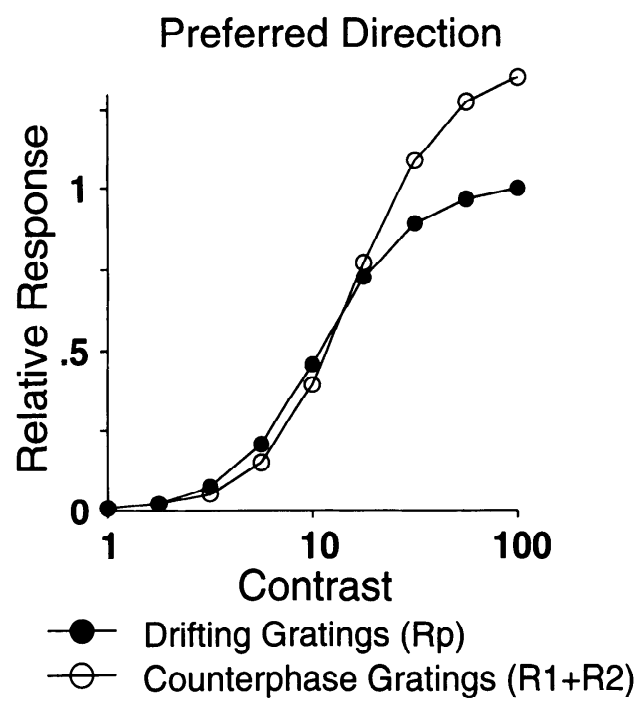

A of rectification. For example, overrectification is like halfwave rectification but with a threshold; the neuron has to reach a certain level of excitation before it will fire action potentials. Albrecht and Geisler (1991) modeled simplecell responses with the use of an exponential nonlinearity in which they allow the exponent to vary from one cell to the next. The normalization model adopted in this paper uses half-squaring, that is, half-wave rectification followed by squaring.

These nonlinear functions (half-wave rectification, overrectification, half-squaring, and exponentiation with a power other than 2) are closely related. We can express them all as special cases of the following function

$$
f(x)=\lfloor x-T\rfloor^{n}
$$

where $T$ and $n>0$ are constants. Half-squaring corresponds to the case in which $n=2$ and $T=0$. Half-wave rectification corresponds to the case in which $n=1$ and $T=0$. Overrectification corresponds to the case in which $n=1$ and $T>0$. Albrecht and Geisler's exponential nonlinearity corresponds to the case in which $T=0$ and $n$ is allowed to vary from one cell to the next.

\section{Non-preferred Direction}

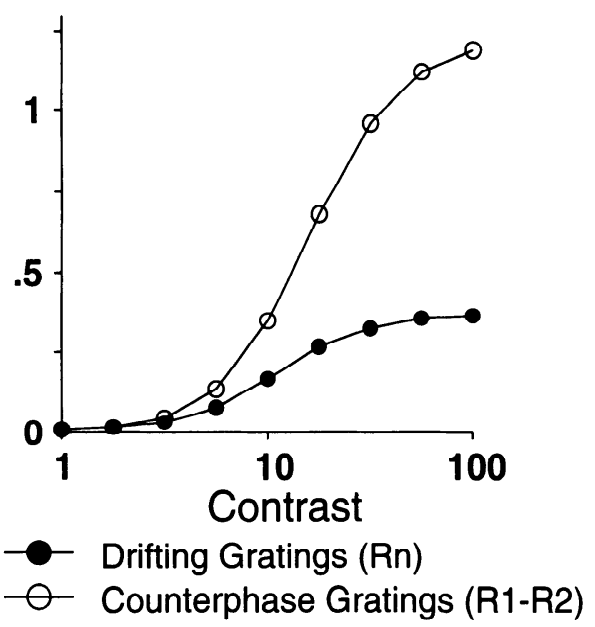

$B$

FIG. 12. Comparison of model cell's response to counterphase and drifting gratings (preferred spatial frequency and orientation), as a function of contrast. $A$ : response to gratings drifting in the preferred direction $(\bullet)$ and predictions of preferred response computed from counterphase data $(0) . B$ : response to gratings drifting in the nonpreferred direction $(\bullet)$ and predictions computed from counterphase data $(0)$. 


\section{Preferred Direction}

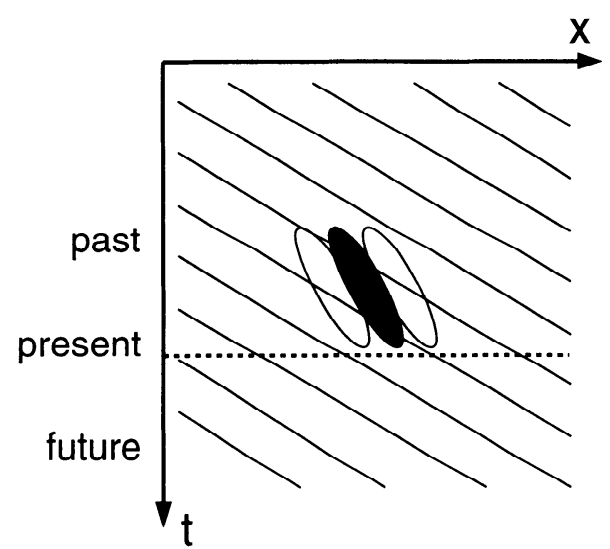

A
Non-preferred Direction

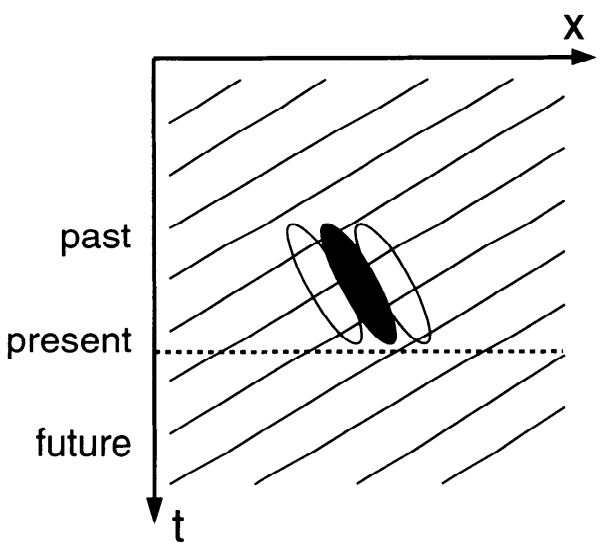

B
FIG. 13. Illustration of spatiotemporal linear cell responding to drifting grating stimuli. $A$ : stimulus drifting in the preferred direction. $B$ : stimulus drifting in the nonpreferred direction. Response to the nonpreferred direction is 0 , i.e., the direction index is 1 .
In this paper we have fixed the exponent at 2 , and we have fixed the threshold at 0 , but they could in principle be allowed to vary. Obviously, adding these two parameters to the model would allow more flexibility in fitting physiological data.

There are, on the other hand, two theoretical reasons for using half-squaring. First, squaring is a useful computation. Several algorithms have been proposed for computing velocity from the squared responses of a set of spatiotemporal linear operators (Adelson and Bergen 1986; Heeger 1987; Heeger and Simoncelli 1993; Grzywacz and Yuille 1990; Simoncelli and Adelson 1991). Second, squaring makes the model analytically tractable (see APPENDIX A). This allows us to derive closed form expressions for fitting physiological data.

What would be the consequences of replacing half-squaring with a more general non-linearity with variable threshold and exponent parameters? We would gain flexibility in fitting data, but we would loose the ability to study the model's behavior analytically. In all likelihood, the normalization model (with or without variable threshold and exponent parameters) is only an approximation to the behavior of real simple cells. It will be a useful model if it proves to be a reasonably good approximation. It will be all the more useful if it is analytically tractable.

\section{Why feedback}

The normalized, half-squared, linear model is similar to a model recently proposed by Albrecht and Geisler (1991). The main difference is that Albrecht and Geisler (1991) use a feed-forward gain control mechanism instead of feedback normalization advocated in this and other papers (Carandini and Heeger 1993; Heeger 1991, 1992a,b).

There are three empirical results that favor the feedback model. First, the feed-forward model predicts that normalization would be evident in geniculate cell responses. This appears not to be the case (Bonds 1989).

Second, the two models (feed-forward and feedback) predict different dynamic behavior. Tolhurst et al. (1980) found that simple cells respond with an initial transient burst of activity when a stimulus first appears. The feed-forward model does not predict this behavior.

The feedback model, on the other hand, behaves simi- larly to real simple cells; it takes some time after a stimulus first appears before the model cells are fully normalized. The initial transient burst of activity is evident in Fig. 5. The dynamics of the feedback network are derived in APPENDIX A. One particularly interesting observation is that the amount of time it takes to reach steady state depends on the stimulus. For high-contrast stimuli, the network reaches steady state very quickly. For low-contrast stimuli, the feedback network is much slower. The model thus predicts that transient behavior in simple cells should depend in a particular way on stimulus contrast.

Third, the feedback model predicts that the normalization signal originates in cortical cells. There is, in fact, evidence that this is true (Bishop et al. 1973; Bonds 1989; DeValois and Tootell 1983; Morrone et al. 1982).

\section{Fitting data}

In this paper, no attempt was made to fit model parameters to the data; the simulations merely point out the qualitative similarities between the behavior of model cells and real cells. We are now in a position to test the model quantitatively. The proper experiment at this time would be to repeat the measurements of drifting and counterphase grating responses using several different contrasts. We could then infer the value of the semisaturation constant $\sigma^{2}$ from the contrast series. Given $\sigma^{2}$, we could analyze the data compensating for the effects of the nonlinearities: first multiply by $\sigma^{2}$ plus the Fourier energy of the stimulus, and then take square roots. If the model is correct, then this analysis (compensating for the nonlinearities) would reveal the underlying linear responses.

\section{APPENDIX A}

This appendix serves several purposes. First, it describes the feedback normalization network and shows that it has the proper steady-state behavior. The network is designed so that for drifting grating stimuli, the steady-state response is given by $E q$. 3. Second, it shows that the feedback signal must be averaged over time to avoid unstable oscillations. Third, it shows that the dynamics of the network (the amount of time it takes to reach steady state) depends on the stimulus.

Figure A 1 illustrates the network. The normalized responses are denoted by $R_{i}(t)$. The feedback signal is denoted by $G(t)$. There are three additional parameters: $\sigma$ is the semi-saturation constant, 


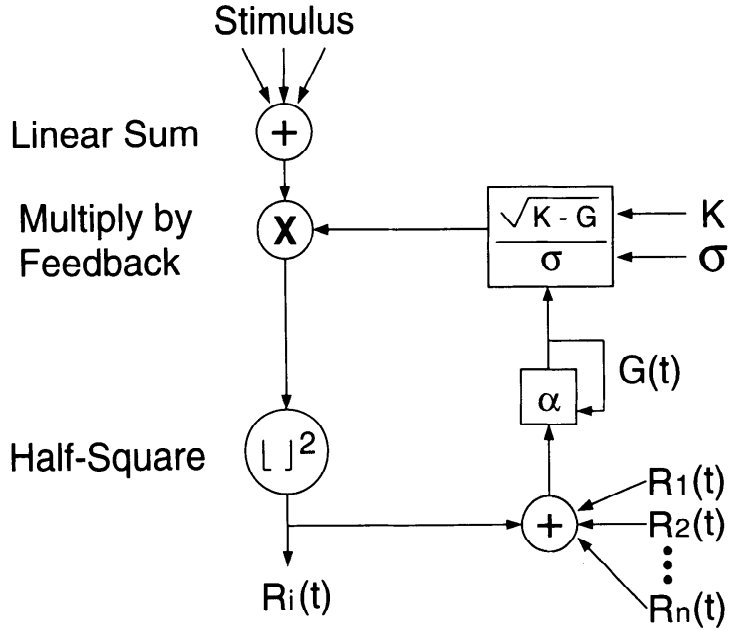

FIG. A1. Diagram of feedback normalization network. $R_{i}(t)$ is the normalized response, $G(t)$ is the feedback signal, $\sigma$ is the semisaturation constant, $\alpha$ specifies the temporal averaging of the feedback, and $K$ determines the maximum attainable response (see text for details).

$\alpha$ specifies the temporal averaging of the feedback, and $K$ determines the maximum attainable response.

The signals in the network are updated over time according to the following equations

$$
\begin{aligned}
& G(t)=(1-\alpha) G(t-1)+\alpha \sum_{j} R_{j}(t) \\
& R_{i}(t)=\left[L_{i}(t) \frac{\sqrt{K-G(t-1)}}{\sigma}\right]^{2}
\end{aligned}
$$

where $L_{i}(t)$ is the linear response defined in $E q$. 1 , where $[x]=$ $\max (x, 0)$ is half-wave rectification, and where it is assumed ${ }^{4}$ that $G(t) \leq K$.

These two equations were used to compute the model simulations. They are written here as a pair of time-difference equations for ease of implementation on a computer. The network could also be expressed in terms of a pair of differential equations, modeling the cortical cell's membrane as an RC circuit whose conductance grows with the activity of all the neurons in the pool (Carandini and Heeger 1993).

The feedback signal $G(t)$ is the time average of the pooled response, $\sum_{j} R_{j}(t)$. In other words, $G(t)$ is computed by convolving $\sum_{j} R_{j}(t)$ with a low-pass filter. The low-pass filter has an exponential impulse response, and $\alpha$ determines its time constant.

The linear response $L_{i}(t)$ is multiplied by the feedback signal, but this actually results in divisive suppression. In the figure the multiplication is shown after linear summation. It could also occur before the summation (just by distributing the multiplication across all of the inputs). In principle, the suppression could be due to corticofugal inhibition from cortical axons onto geniculate cell dendrites, it could be due to presynaptic inhibition (affecting the release of neurotransmitter) from cortical axons onto geniculate axons, it could be due to inhibition from cortical axons onto dendrites of other cortical cells, or it could be some combination of these. As mentioned in the DISCUSSION, however, there is evidence that the suppression is mostly intracortical, i.e., that it originates in cortical cells and that it affects only cortical cells (Bishop et al. 1973; Bonds 1989; DeValois and Tootell 1983; Morrone et al. 1982).

Because we assume that $G(t) \leq K$, we can rewrite $E q$. A2

\footnotetext{
${ }^{4}$ We can ensure that $G(t) \leq K$ by clipping values larger than $K$, i.e., by using $G^{\prime}(t)=\min [G(t), K]$.
}

$$
R_{i}(t)=A_{i}(t) \frac{K-G(t-1)}{\sigma^{2}}
$$

where $A_{i}(t)=\left\lfloor L_{i}(t)\right\rfloor^{2}$ is a half-squared, linear response.

To show that the network has the desired steady-state behavior we rewrite $E q$. Al substituting for $R_{j}(t)$ from $E q$. A3

$$
\tau[G(t)-G(t-1)]=-G(t-1)+G_{s}(t)
$$

where

$$
\tau=\frac{\sigma^{2}}{\alpha\left[\sigma^{2}+\sum_{j} A_{j}(t)\right]}
$$

and

$$
G_{s}(t)=K\left[\frac{\sum_{j} A_{j}(t)}{\sigma^{2}+\sum_{j} A_{j}(t)}\right]
$$

In steady state, $G(t)=G(t-1)$, the left-hand side of $E q$. A4 is zero, and the equation simplifies to $G(t)=G_{s}(t)$. Thus it is clear that $G_{s}(t)$ is the steady-state value for the feedback signal.

The reader may find it odd to talk about $G_{s}(t)$ as a steady-state value given that it is, in general, a time-varying function of $A_{i}(t)$. For drifting grating stimuli, however, $\sum_{j} A_{j}(t)$ is constant over time. The underlying linear weighting functions in the model are designed in quadruples: four cells with the same amplitude response but with phases in steps of $90^{\circ}$. For a drifting sine grating stimulus, the sum of the four responses is proportional to the squared contrast of the stimulus, and it is constant over time (independent of the stimulus phase). The phase independence is a simple consequence of the $90^{\circ}$ phase offsets, analogous to the trigonometric identity: $\sin ^{2}(\theta)+\cos ^{2}(\theta)=1$.

Substituting $G_{s}(t)$ for $G(t-1)$ in $E q$. A 3 gives the response of a model cell, once the network has reached in steady state

$$
R_{i}(t)=K \frac{A_{i}(t)}{\sigma^{2}+\sum_{j} A_{j}(t)}
$$

that is precisely the same as $E q .3$.

From $E q$. A4, we can also analyze the dynamics of the network. The solution to Eq. A4 is a complex exponential function with time constant, $\tau$. From $E q$. A5, it is clear that the time constant depends on the input. For high-contrast stimuli, $\sum_{j} A_{j}(t)$ is large, $\tau$ is small, and the network converges quickly. For low-contrast stimuli, on the other hand, the time constant is longer. Note that the time constant depends on the Fourier energy of the stimulus (proportional to pooled activity of a number of model cells), not on responsiveness of any particular model cell.

If $\tau$ is too small, then the feedback network becomes unstable. In particular, $\tau$ must be $>1 / 2$ to avoid unstable oscillations. In other words, we must ensure that

$$
\alpha<\frac{2 \sigma^{2}}{\sigma^{2}+\sum_{j} A_{j}(t)}
$$

The total activity, $\sum_{j} A_{j}(t)$, is bounded, because it is proportional to the Fourier energy of the visual stimulus. Let $A_{0}$ be the largest possible value for $\sum_{j} A_{j}(t)$. Likewise, let $\sigma_{0}$ be the smallest possible value for $\sigma$ (because we choose $\sigma$ in the model we can guarantee that it will never be smaller than $\sigma_{0}$ ). Then we choose $\alpha$ as follows

$$
\alpha<\frac{2 \sigma_{0}^{2}}{\sigma_{0}^{2}+A_{0}}
$$

As long as $\sigma \geq \sigma_{0}$ and $\sum_{j} A_{j}(t) \leq A_{0}$, then $\tau$ will be $>1 / 2$. For the simulations reported in this paper, the parameters were chosen as follows: $K=1$ so that $\sum_{j} A_{j}(t) \leq 1, \sigma=0.1$, and $\alpha=0.01$ to satisfy the above constraint. 


\section{APPENDIX B}

This appendix derives expressions approximating the model's behavior in Figs. 9-12. This appendix shows 1) that the linear prediction from counterphase data underestimates the direction index (computed from drifting grating responses); 2) that the linear prediction from counterphase data overestimates the response to gratings drifting in the nonpreferred direction; and 3 ) that, for an appropriate choice of contrast, the linear prediction from counterphase data correctly estimates the response to gratings drifting in the preferred direction.

Three approximations are made in deriving these results. First, the ellipse fitting procedure is ignored, and it is assumed that we have measured counterphase grating responses for the spatial phases giving the maximum and minimum responses. Second, the initial transient burst of activity is ignored. Third, it is assumed that the feedback normalization signal, $\sum_{j} R_{j}(t)$ in $E q$. Al, is constant over time. The normalization signal is indeed constant over time for a drifting grating stimulus ( see APPENDIX A). For a counterphase grating stimulus, however, the normalization signal oscillates over time. The magnitude of the oscillations depends on the extent of temporal averaging (smaller $\alpha$ in $E q$. Al yields more averaging).

In the following derivations, the responses of the underlying linear operator are denoted by $R_{\mathrm{p}}, R_{\mathrm{n}}, R_{1}$, and $R_{2}$. In particular, $R_{\mathrm{p}}$ is the magnitude of the fundamental Fourier component of the response for a grating drifting in the preferred direction, and $R_{\mathrm{n}}$ is the response magnitude for a grating drifting in the nonpreferred direction. $R_{1}$ and $R_{2}$ are the magnitudes for counterphase gratings with spatial phases eliciting the largest and smallest (respectively) responses. Likewise, the response magnitudes of the model cell (normalized, half-squared, linear operator) are denoted by $\bar{R}_{\mathrm{p}}$, $\bar{R}_{\mathrm{n}}, \bar{R}_{1}$, and $\bar{R}_{2}$.

The response of a model cell is given by $E q .3$, rewritten here as

$$
R_{i}(t) \approx K \frac{A_{i}(t)}{\sigma_{1}^{2}+k_{1}^{2} E}
$$

where $\sigma_{1}^{2}$ is the semisaturation constant and $K$ and $k_{1}$ are constants. $A_{i}(t)$ is the response of an unnormalized, half-squared, linear operator. $E$ is the Fourier energy of the stimulus (because the linear weighting functions tile the spatiotemporal frequency domain, the feedback normalization signal is proportional to the Fourier energy of the stimulus). This expression is an approximation to the actual model behavior. It is exact for drifting grating stimuli, because for those stimuli the feedback normalization signal, $\sum_{j} R_{j}(t)$ in $E q$. $\mathrm{A} l$, is constant over time.

The following statements are true of the underlying linear response magnitudes

$$
\begin{aligned}
& R_{\mathrm{p}}=R_{1}+R_{2} \\
& R_{\mathrm{n}}=R_{1}-R_{2} \\
& R_{1}=\left(R_{\mathrm{p}}+R_{\mathrm{n}}\right) / 2 \\
& R_{2}=\left(R_{\mathrm{p}}-R_{\mathrm{n}}\right) / 2
\end{aligned}
$$

These equalities are used in the derivations below.

We can write the normalized response magnitudes in terms of the underlying linear response magnitudes. For example

$$
\bar{R}_{\mathrm{p}}=k_{2} \frac{\left(R_{\mathrm{p}}\right)^{2}}{\sigma_{1}^{2}+k_{1}^{2} c^{2}}=k \frac{\left(R_{\mathrm{p}}\right)^{2}}{\sigma^{2}+c^{2}}
$$

where $c$ is Michelson contrast; $k, k_{1}$, and $k_{2}$ are constants; $\sigma=$ $\sigma_{1} / k_{1}$; and $k=k_{2} / k_{1}^{2}$. The denominator depends on the semisaturation constant and on the Fourier energy of the stimulus. A drifting grating with Michelson contrast $c$ has Fourier energy equal to $c^{2}$.
The other three normalized responses are written similarly

$$
\begin{aligned}
& \bar{R}_{\mathrm{n}}=k \frac{\left(R_{\mathrm{n}}\right)^{2}}{\sigma^{2}+c^{2}} \\
& \bar{R}_{1}=k \frac{\left(R_{1}\right)^{2}}{\sigma^{2}+c^{2} / 2} \\
& \bar{R}_{2}=k \frac{\left(R_{2}\right)^{2}}{\sigma^{2}+c^{2} / 2}
\end{aligned}
$$

The denominators of the expressions for $\bar{R}_{1}$ and $\bar{R}_{2}$ have $c^{2} / 2$, whereas the denominators of the expressions for $\bar{R}_{\mathrm{p}}$ and $\bar{R}_{\mathrm{n}}$ have $c^{2}$. Counterphase gratings and drifting gratings of equal contrast have different Fourier energy.

We now show that the linear prediction from counterphase data underestimates the direction index. The direction index (computed from drifting grating responses) for a model cell is $\left(\bar{R}_{\mathrm{p}}-\right.$ $\left.\bar{R}_{\mathrm{n}}\right) /\left(\bar{R}_{\mathrm{p}}+\bar{R}_{\mathrm{n}}\right)$. The linear prediction from counterphase data is $\bar{R}_{2} / \bar{R}_{1}$. The first expression is always greater than or equal to the latter

$$
\begin{aligned}
\frac{\bar{R}_{\mathrm{p}}-\bar{R}_{\mathrm{n}}}{\bar{R}_{\mathrm{p}}+\bar{R}_{\mathrm{n}}} & =\frac{R_{\mathrm{p}}^{2}-R_{\mathrm{n}}^{2}}{R_{\mathrm{p}}^{2}+R_{\mathrm{n}}^{2}} \\
& =\frac{\left(R_{\mathrm{p}}-R_{\mathrm{n}}\right)\left(R_{\mathrm{p}}+R_{\mathrm{n}}\right)}{R_{\mathrm{p}}^{2}+R_{\mathrm{n}}^{2}} \\
& \geq \frac{\left(R_{\mathrm{p}}-R_{\mathrm{n}}\right)^{2}}{R_{\mathrm{p}}^{2}+R_{\mathrm{n}}^{2}} \quad \text { since } R_{\mathrm{p}} \geq R_{\mathrm{n}} \geq 0 \\
& \geq \frac{\left(R_{\mathrm{p}}-R_{\mathrm{n}}\right)^{2}}{\left(R_{\mathrm{p}}+R_{\mathrm{n}}\right)^{2}} \quad \text { since } R_{\mathrm{p}}, R_{\mathrm{n}} \geq 0 \\
& =R_{2}^{2} / R_{1}^{2} \\
& =\bar{R}_{2} / \bar{R}_{1}
\end{aligned}
$$

Next we show that the linear prediction from counterphase data overestimates the response to a grating drifting in the nonpreferred direction. The response to a grating drifting in the nonpreferred direction is $\bar{R}_{\mathrm{n}}$. The linear prediction from counterphase data is $\bar{R}_{1}-\bar{R}_{2}$. The first expression is always less than or equal to the latter

$$
\begin{aligned}
\bar{R}_{\mathrm{n}} & =k \frac{R_{\mathrm{n}}^{2}}{\sigma^{2}+c^{2}} \\
& =k \frac{\left(R_{1}-R_{2}\right)^{2}}{\sigma^{2}+c^{2}} \\
& \leq k \frac{\left(R_{1}-R_{2}\right)\left(R_{1}+R_{2}\right)}{\sigma^{2}+c^{2}} \quad \text { since } R_{1} \geq R_{2} \geq 0 \\
& =k \frac{R_{1}^{2}-R_{2}^{2}}{\sigma^{2}+c^{2}} \\
& \leq k \frac{R_{1}^{2}-R_{2}^{2}}{\sigma^{2}+c^{2} / 2} \\
& =\bar{R}_{1}-\bar{R}_{2}
\end{aligned}
$$

Next we show that for an appropriate choice of contrast, the linear prediction from counterphase data correctly estimates the response to a grating drifting in the preferred direction. Two results are derived: 1 ) when the contrast is relatively large, the linear prediction from counterphase data overestimates the preferred response (evident in Fig. 12 A); and 2) when the contrast is relatively small, the linear prediction underestimates the preferred response (also evident in Fig. 12 A) . For an appropriate choice of contrast, in between these two extremes, the linear prediction is close to the preferred response (as in Fig. 11).

The response to a grating drifting in the preferred direction is $\bar{R}_{\mathrm{p}}$. The linear prediction from counterphase data is $\bar{R}_{1}+\bar{R}_{2}$ 


$$
\begin{aligned}
\bar{R}_{1}+\bar{R}_{2} & =k \frac{R_{1}^{2}+R_{2}^{2}}{\sigma^{2}+c^{2} / 2} \\
& =k \frac{2\left(R_{1}^{2}+R_{2}^{2}\right)}{2 \sigma^{2}+c^{2}} \\
& =k \frac{\left(R_{1}^{2}+R_{2}^{2}\right)+\left(R_{1}^{2}-R_{2}^{2}\right)}{\sigma^{2}+c^{2}}\left(\frac{\sigma^{2}+c^{2}}{2 \sigma^{2}+c^{2}}\right) \\
& =\left(\bar{R}_{\mathrm{p}}+\bar{R}_{\mathrm{n}}\right)\left(\frac{\sigma^{2}+c^{2}}{2 \sigma^{2}+c^{2}}\right) \\
& =\bar{R}_{\mathrm{p}}\left[1+\left(\bar{R}_{0} / \bar{R}_{\mathrm{p}}\right)\right]\left(\frac{\sigma^{2}+c^{2}}{2 \sigma^{2}+c^{2}}\right)
\end{aligned}
$$

The counterphase prediction overestimates the preferred response $\bar{R}_{1}+\bar{R}_{2}>\bar{R}_{\mathrm{p}}$ if and only if the following condition holds

$$
\left[1+\left(\bar{R}_{\mathrm{n}} / \bar{R}_{\mathrm{p}}\right)\right]\left(\frac{\sigma^{2}+c^{2}}{2 \sigma^{2}+c^{2}}\right)>1
$$

i.e.,

$$
c^{2}>\sigma^{2}\left(\frac{\bar{R}_{\mathrm{p}}-\bar{R}_{\mathrm{n}}}{\bar{R}_{\mathrm{n}}}\right)
$$

Likewise, the counterphase prediction underestimates the preferred response $\bar{R}_{1}+\bar{R}_{2}<\bar{R}_{\mathrm{p}}$ if and only if

$$
c^{2}<\sigma^{2}\left(\frac{\bar{R}_{\mathrm{p}}-\bar{R}_{\mathrm{n}}}{\bar{R}_{\mathrm{n}}}\right)
$$

Special thanks to E. Simoncelli for helping to design the spatiotemporal linear weighting functions used for the simulations in this paper, to E. J. Chichilnisky for help with APPENDIX B, to M. Shadlen for helpful comments on the manuscript, and to C. Reid and D. Tolhurst for providing empirical data.

This research was supported by National Institute of Mental Health Grant 1-R29-MH-50228-01, National Aeronautics and Space Administration-Ames Grant NCC2-307, and NASA RTOP 506-71-51.

Received 16 November 1992; accepted in final form 13 July 1993.

\section{REFERENCES}

ADELSON, E. H. AND BERGEN, J. R. Spatiotemporal energy models for the perception of motion. J. Opt. Soc. Am. A 2: 284-299, 1985.

ADELSON, E. H. AND BERGEN, J. R. The extraction of spatio-temporal energy in human and machine vision. Proc. IEEE Workshop on Motion: Representation and Analysis, Charleston, SC, 1986, p. 151-156.

AlBRECHT, D. G. AND GeISLER, W. S. Motion sensitivity and the contrastresponse function of simple cells in the visual cortex. Visual Neurosci. 7: 531-546, 1991.

AlbReCht, D. G. AND HAMilton, D. B. Striate cortex of monkey and cat: contrast response function. J. Neurophysiol. 48: 217-237, 1982.

ANDREwS, B. W. AND Pollen, D. A. Relationship between spatial frequency selectivity and receptive field profile of simple cells. J. Physiol. Lond. 287: 163-176, 1979.

BishOP, P. O., COOMBS, J. S., AND HeNRY, G. H. Receptive fields of simple cells in the cat striate cortex. J. Physiol. Lond. 231: 31-60, 1973.

BoNDS, A. B. Role of inhibition in the specification of orientation selectivity of cells in the cat striate cortex. Visual Neurosci. 2: 41-55, 1989.

BURR, D. C., Ross, J., AND MORRONE, M. C. Seeing objects in motion. Proc. R. Soc. Lond. B Biol. Sci. 227: 249-265, 1986.

Campbell, F. W., Cleland, B. G., Cooper, G. F., and Enroth-CuGELL, C. The angular selectivity of visual cortical cells to moving gratings. J. Physiol. Lond. 198: 237-250, 1968.

CAMPbell, F. W., COOPER, G. F., AND ENROTh-Cugell, C. The spatial selectivity of visual cells of the cat. J. Physiol. Lond. 203: 223-235, 1969.

CARANDINI, M. AND HeEgER, D. J. Normalization with shunting inhibition explains simple cell response phase and integration time (Abstract). Invest. Ophthalmol. Visual Sci. Suppl. 34: 907, 1993.

DEAN, A. F. The contrast-dependence of direction-selectivity (Abstract). J. Physiol. Lond. 303: 38P-39P, 1980.

DEAN, A. F. The relationship between response amplitude and contrast for cat striate cortical neurones. J. Physiol. Lond. 318: 413-427, 1981.
DEAN, A. F. AND TOLHURST, D. J. Factors influencing the temporal phase of response to bar and grating stimuli for simple cells in the cat striate cortex. Exp. Brain Res. 62: 143-151, 1986.

DeAngelis, G. C., Ohzawa, I., and Freeman, R. D. The spatiotemporal organization of simple cell-receptive fields in the cat's striate cortex. I. General characteristics and postnatal development. J. Neurophysiol. 69: 1091-1117, 1993a.

DeANGelis, G. C., OHZAWA, I., AND FreEMAN, R. D. The spatiotemporal organization of simple cell-receptive fields in the cat's striate cortex. II. Linearity of temporal and spatial summation. J. Neurophysiol. 69: 1118-1135, 1993b.

DeVAloIS, K. AND TOOTELl, R. Spatial-frequency-specific inhibition in cat striate cortex cells. J. Physiol. Lond. 336: 359-376, 1983.

FAHLE, M. AND PoGGIO, T. Visual hyperacuity: spatiotemporal interpolation in human vision. Proc. R. Soc. Lond. B Biol. Sci. 213: 451-477, 1981.

GrzywaCZ, N. M. AND YUILLE, A. L. A model for the estimate of local image velocity by cells in the visual cortex. Proc. R. Soc. Lond. B Biol. Sci. 239: 129-161, 1990.

HeEger, D. J. Model for the extraction of image flow. J. Opt. Soc. Am. A 4: 1455-1471, 1987.

HeEger, D. J. Nonlinear model of cat striate physiology. Soc. Neurosci. Abstr. 16: 229, 1990.

HEEGER, D. J. Nonlinear model of neural responses in cat visual cortex. In: Computational Models of Visual Processing, edited by M. Landy and J. A. Movshon. Cambridge, MA: MIT Press, 1991, p. 119-133.

HeEger, D. J. Normalization of cell responses in cat striate cortex. Visual Neurosci. 9: 181-198, 1992a.

HEEGER, D. J. Half-squaring in responses of cat simple cells. Visual Neurosci. 9: 427-443, $1992 \mathrm{~b}$.

HEEGER, D. J. Modeling simple cell direction selectivity with normalized, half-squared, linear operators (Abstract). Invest. Ophthalmol. Visual Sci. Suppl. 33: 953, 1992c.

HEEger, D. J. AND AdELSON, E. H. Nonlinear model of cat striate cortex (Abstract). Optics News 15: A-42, 1989.

HeEger, D. J. AND SimonCElli, E. P. Model of visual motion sensing. In: Spatial Vision in IIumans and Robots, edited by L. Harris and M. Jenkins. New York: Cambridge Univ. Press, 1993, p. 367-392.

Holub, R. A. AND MORTON-Gibson, M. Response of visual cortical neurons of the cat to moving sinusoidal gratings: response-contrast functions and spatiotemporal interactions. J. Neurophysiol. 46: 1244-1259, 1981.

HubEL, D. AND Wiesel, T. Receptive fields, binocular interaction, and functional architecture in the cat's visual cortex. J. Physiol. Lond. 160: 106-154, 1962.

KulikowsKi, J. J. AND BISHOP, P. O. Linear analysis of the response of simple cells in the cat visual cortex. Exp. Brain Res. 44: 386-400, 1981.

Li, C. AND CREUTzFeldT, O. The representation of contrast and other stimulus parameters by single neurons in area 17 of the cat. Pfluegers Arch. 401: 304-314, 1984.

MAFFEI, L. AND FIORENTINI, A. The visual cortex as a spatial frequency analyzer. Vision Res. 13: 1255-1267, 1973.

MCLean, J. AND PALmer, L. A. Contribution of linear spatiotemporal receptive field structure to velocity selectivity of simple cells in area 17 of cat. Vision Res. 29: 675-679, 1989.

Morrone, M. C., BURR, D. C., AND MAFFEI, L. Functional implications of cross-orientation inhibition of cortical visual cells. Proc. R. Soc. Lond. B Biol. Sci. 216: 335-354, 1982.

Movshon, J. A., Thompson, I. D., AND Tolhurst, D. J. Spatial summation in the receptive fields of simple cells in the cat's striate cortex. $J$. Physiol. Lond. 283: 53-77, 1978.

REID, R. C., SOODAK, R. E., AND ShaPley, R. M. Linear mechanisms of directional selectivity in simple cells of cat striate cortex. Proc. Natl. Acad. Sci. USA 84: 8740-8744, 1987.

ReID, R. C., SOODAK, R. E., AND SHAPley, R. M. Directional selectivity and spatiotemporal structure of receptive fields of simple cells in cat striate cortex. J. Neurophysiol. 66: 505-529, 1991.

RoBSON, J. G. Linear and nonlinear operations in the visual system (Abstract). Invest. Ophthalmol. Visual Sci. Suppl. 29: 117, 1988.

SCLAR, G. AND FREEMAN, R. D. Orientation selectivity of the cat's striate cortex is invariant with stimulus contrast. Exp. Brain Res. 46: 457-461, 1982.

ShaPley, R., ReID, R. C., AND SoodaK, R. Spatiotemporal receptive 
fields and direction selectivity. In: Computational Models of Visual Processing, edited by M. Landy and J. A. Movshon. Cambridge, MA: MIT Press, 1991, p. 109-118.

SimONCELli, E. P. AND Adelson, E. H. Relationship between gradient, spatio-temporal energy, and regression models for motion perception (Abstract). Invest. Ophthalmol. Visual Sci. Suppl. 32: 893, 1991.

TOLHURST, D. J. AND DEAN, A. F. Evaluation of a linear model of directional selectivity in simple cells of the cat's striate cortex. Visual Neurosci. 6: 421-428, 1991.
Tolhurst, D. J., Walker, N. S., Thompson, I. D., AND Dean, A. F. Nonlinearities of temporal summation in neurones in area 17 of the cat. Exp. Brain Res. 38: 431-435, 1980.

WATSON, A. B. AND Ahumada, A. J. A look at motion in the frequency domain. In: Motion: Perception and Representation, edited by J. K. Tsotsos. New York: Association for Computing Machinery, 1983, p. $1-10$.

Watson, A. B. AND Ahumada, A. J. Model of human visual-motion sensing. J. Opt. Soc. Am. A 2: 322-342, 1985. 